Chirurgia (2020) 115: 191-207

No. 2, March - April

Copyright@ Celsius

http://dx.doi.org/10.21614/chirurgia.115.2.191

\title{
What are the Specifics of Biliary Surgery in Cirrhotic Patients?
}

\author{
François Cauchy ${ }^{1}$, Eric Vibert ${ }^{2}$, Louise Barbier ${ }^{3}$ \\ 'Department of HPB Surgery and liver transplantation, Beaujon Hospital, Clichy, France \\ Assistance Publique Hôpitaux de Paris and Université de Paris, Paris, France \\ 2Department of HPB Surgery and liver transplantation, Paul Brousse Hospital, Villejuif \\ Assistance Publique Hôpitaux de Paris and Université Paris XI, Paris, France \\ ${ }^{3}$ Department of digestive, endocrine, HPB surgery and liver transplantation, Trousseau University Hospital, Tours, France
}

Corresponding author:

François Cauchy, MD Department of HPB surgery and liver transplantation, Beaujon Hospital, Clichy, France E-mail: francois.cauchy@aphp.fr

\section{Rezumat}

Care sunt specificațiile chirurgiei biliare la pacienții cirotici?

Obiectivul acestei lucrări a fost să trecem în revistă întreaga literatură chirurgicală biliară cu scopul de a defini în mod cât mai adecvat indicațiile chirurgicale şi specificul managementului lor terapeutic. Au fost luate în considerare publicațile din PubMed şi Google Scholar în limbile franceză şi engleză din perioada 1995 - august 2015.

Cuvinte cheie: ciroză, chirurgie biliară, colecistită, complicații postoperatorii

\section{Abstract}

This work's objective was to review the literature on biliary surgery in order to best define the surgical indications and the specifics of their management. A review of the literature from 1995 to August 2015 was conducted in Pubmed and Google Scholar.

Key words: cirrhosis, biliary surgery, cholecystitis, postoperative complications 


\section{Introduction}

This chapter reports the results of surgical studies in cirrhotic patients requiring gallbladder and extrahepatic bile duct surgery. The vast majority of these studies concerned lithiasic pathology, in particular vesicular, and focused on the comparison of the different surgical approaches in this context. In contrast, very few recent studies have addressed the lithiasic pathology of the main bile duct in cirrhotic patients and even fewer have focused specifically on surgery of the extrahepatic bile ducts in cirrhotic patients in a non-lithiasic context.

\section{Performed Research}

A review of the literature from 1995 to August 2015 was conducted in Pubmed and Google Scholar, using the following keywords: "cirrhosis" in combination with one or more of the following terms: "extra-hepatic bile duct surgery; cholecystitis; cholecystectomy; laparoscopic cholecystectomy; sub-total cholecystectomy; cholecystostomy; cholangitis; endoscopic retrograde cholangiopancreatography; gallbladder stones; cholelithiasis; choledocolithiasis; common bile duct stones; common bile duct resection; and hepaticojejunostomy". Retrospective studies journals and books were also included. The articles were then analyzed and grouped according to their themes: lithiasic pathology (vesicular or main bile duct) and nonlithiasic pathology.

\section{Surgical Indications}

Reminder on the biliary pathology of the cirrhotic patient

Incidence and physiology of cholelithiasis in the cirrhotic patient

Gallstones, especially gallbladder stones, are common in patients with cirrhosis with an incidence of 9.5-29.4\%, compared to 5.2-12.8\% in patients without cirrhosis $(1,2)$. Their prevalence is increasing with age, female gender, thickness of the gallbladder wall (3) and severity of complementary liver disease (4). These stones are pigmentary in nature in 50 to $85 \%$ (4). This high rate is linked to a bile unconjugated bilirubin concentration increase in cirrhotic patients and seems to be due to various favourable factors such as: 1) the existence of a low ratio between bile acids and unconjugated bilirubin (5); 2) induction of an entero cycle -hepatic unconjugated bilirubin favoured by chronic alcohol consumption and low protein diets; 3) chronic haemolysis linked to hypersplenism (6); and 4) biliary stasis secondary to vesicular hypotonia. Nevertheless, the clear predominance of pigmentary stones, by nature brittle and small, seems to explain the relatively low incidence of complications linked to the presence of vesicular lithiasis in cirrhotic patients. On the other hand, it is important to note that the existence of cirrhosis linked to a NAFLD (Non Alcoholic Fatty liver disease) seems to favour the development of cholesterol stones (7-9) and that it will thus probably be expected to observe an increase in the incidence and type of complications associated with these stones in the coming decades.

\section{Complications of cholelithiasis in cirrhotic patients}

Gallbladder stones are usually asymptomatic in cirrhotic patients and are more likely to be detected during follow-up ultrasound examinations. Historically, cirrhotic patients had higher rates of cholecystectomy than the general population $(10,11)$. In a retrospective study (11), it was nevertheless pointed out that only $62 \%$ of cholecystectomized cirrhotic patients had experienced an episode of abdominal pain of biliary origin. This result could be explained by the absence of a preoperative diagnosis of cirrhosis, leading to a false interpretation of abnormal liver function tests in the context of vesicular lithiasis and of vesicular lithiasis complicated episodes.

The frequency of severe complications associated with gallbladder stones in cirrhotic patients has been evaluated in a few previous studies preceding the popularization of the laparoscopic approach in the management of 
gallstone disease. This risk of complications seems to vary from 0 to $22 \%(6,12,13)$. It should be noted that only one study had reported a complication rate of $0 \%$ but was burdened with a loss of sight rate which did not allow a solid conclusion (12). The most commonly reported complications are chronic cholecystitis (55\%) followed by acute cholecystitis $(45 \%)$ and cholangitis $(<5 \%)$.

A 2003 meta-analysis (14) reported that the most frequent indications of laparoscopic cholecystectomy in the cirrhotic patient were dominated by hepatic colic in $54 \%$ of cases, followed by (acute or chronic) lithiasic cholecystitis in $22 \%$ of cases, vesicular asymptomatic lithiasis in $16 \%$ of cases, acute biliary pancreatitis in $5 \%$ of cases, and alithiasic cholecystitis and, respectively, lithiasis of the main bile duct in less than $1 \%$ of cases.

Nowadays, the Tokyo consensus conference, although not specific to cirrhotic patients, is the only recommendation available to stratify the severity (Tables 1 and 2) and formalize acute cholecystitis (15) and acute cholangitis (16) management in cirrhotic patients. While some of the consensus conference's severity criteria incorporate certain parameters common to those found in cirrhotic patients, there are, however, no studies validating the applicability of these recommendations in this specific context. Thus, while it appears obvious that the severity of the underlying liver disease plays a predominant role in the severity of acute cholecystitis and acute cholangitis in cirrhotic patients, no recommendation has specifically included this parameter in the strategy for taking care of patients suffering from these pathologies.

\section{Technical Specificities}

\section{Cholecystectomy}

In total, 34 studies published between 2000 and 2015 were analysed, including 2 meta- $^{-}$ analyses of randomized controlled trials $(17,18), 4$ prospective randomized trials (1922), 3 comparative retrospective studies (2325), 19 non-comparative retrospective studies (26-41), 1 meta-analysis of retrospective studies (14) and 4 reviews of retrospective studies (42-45) (Table 3). No study was specifically interested in open cholecystectomy, for which it therefore seems obsolete to

Table 1. Criteria for severity of acute cholecystitis

\begin{tabular}{|c|c|}
\hline \multicolumn{2}{|c|}{ Grade III: Severe acute cholecystitis } \\
\hline 1. Cardio-circulatory & Hypotension requiring treatment with Dopamine $\geq 5 \mu \mathrm{g} / \mathrm{kg}$ per min, or norepinephrine at any dosage \\
\hline 2. Neurological & Altered consciousness \\
\hline 3. Respiratory & $\mathrm{PaO} 2 / \mathrm{Fi} 02<300$ \\
\hline 4. Renal & Oliguria, Serum Creatinine $>2 \mathrm{mg} / \mathrm{dl}$ \\
\hline 5. Hepatic & TP-INR $>1.5 \mathrm{~N}$ \\
\hline 6. Hematologic & Platelet count $<100.000 / \mathrm{mm}^{3}$ \\
\hline \multicolumn{2}{|c|}{ Grade II: Moderate acute cholecystitis } \\
\hline \multicolumn{2}{|c|}{ Defined as acute cholangitis associated with at least two of the following conditions: } \\
\hline \multicolumn{2}{|c|}{ Hyperleukocytosis (> 18.000/mm²) } \\
\hline \multicolumn{2}{|c|}{ Painful palpable mass or resistance in right hypochondrium } \\
\hline \multicolumn{2}{|c|}{ Duration of symptoms $\geq 72$ hours } \\
\hline \multicolumn{2}{|c|}{ Marked local inflammation (gangrenous or emphysematous cholecystitis, perivesicular abscess, hepatic abscess, biliary peritonitis) } \\
\hline \multicolumn{2}{|c|}{ Grade I: Uncomplicated acute cholecystitis } \\
\hline \multicolumn{2}{|c|}{ Defined as not meeting any of the criteria for grades II and III } \\
\hline $\begin{array}{l}\text { Early surgical manag } \\
\text { with rapid reassessn } \\
\text { delayed surgery) is } \\
\text { management combin }\end{array}$ & $\begin{array}{l}\text { Inder cover of preoperative antibiotic therapy is recommended for grade I cholecystitis. First antibiotic therapy } \\
\text { order to decide on secondary management (vesicular drainage or continuation of conservative treatment then } \\
\text { ended for grade II cholecystitis. Early surgery can also be offered in expert centres. Finally, resuscitation } \\
\text { antibiotic therapy and vesicular drainage is recommended for grade III cholecystitis. }\end{array}$ \\
\hline
\end{tabular}


Table 2. Criteria for severity of acute cholangitis

Grade III: Severe acute cholangitis

Defined as acute cholangitis associated with at least one of the following dysfunctions:

1. Cardio-circulatory $\quad$ Hypotension requiring treatment with Dopamine $\geq 5 \mu \mathrm{g} / \mathrm{kg}$ per min, or norepinephrine at any dosage

2. Neurological Altered consciousness

3. Respiratory $\quad \mathrm{PaO2} / \mathrm{FiO} 2<300$

4. Renal Oliguria, Serum Creatinine $>2 \mathrm{mg} / \mathrm{dl}$

5. Hepatic TP-INR $>1.5 \mathrm{~N}$

6. Hematologic Platelet count $<100.000 / \mathrm{mm} 3$

Grade II: Moderate acute cholangitis

Defined as acute cholangitis associated with at least two of the following conditions:

1. Hyperleukocytosis $\left(>12.000 / \mathrm{mm}^{3}\right)$ or leukopenia $\left(<4000 / \mathrm{mm}^{3}\right)$

2. Fever $\geq 39^{\circ} \mathrm{C}$

3. Age $\geq 75$ years old

4. Total bilirubinaemia $\geq 5 \mathrm{mg} / \mathrm{dL}(\geq 85 \mu \mathrm{mol} / \mathrm{l})$

5. Hypoalbuminemia $(<0.7 \mathrm{~N})$

Grade I: Uncomplicated acute cholangitis

Defined as not meeting any of the criteria for grades II and III

Antibiotic treatment followed by biliary drainage and treatment of aetiology is recommended for grade I angiocholites Antibiotic treatment with rapid biliary drainage followed by treatment of aetiology is recommended for angiocholites grade II. Antibiotic treatment with emergency biliary drainage followed by treatment of the aetiology is recommended for grade II angiocholites. Resuscitative care with antibiotic therapy and emergency biliary drainage followed by treatment of the aetiology is recommended for grade III angiocholites.

formalize practical recommendations based on 13 retrospective studies clearly reported the recent literature.

indications for cholecystectomy in cirrhotic

In our review of the literature, 14 articles patients (Annex 1). The total number of including a prospective randomized trial and patients who could be analysed was 896 . The

Table 3. Level of evidence from studies reporting cholecystectomy results in cirrhotic patients between 2000 and 2015

\begin{tabular}{|c|c|c|}
\hline Level of Scientific Evidence Provided by the Literature & Before selection & After selection \\
\hline \multicolumn{3}{|l|}{ Level 1} \\
\hline High-power randomized controlled trials & 3 & 0 \\
\hline Meta-analysis of randomized controlled trials & 2 & 2 \\
\hline \multicolumn{3}{|l|}{ Decision analysis based on well-conducted data } \\
\hline \multicolumn{3}{|l|}{ Level 2} \\
\hline Low-power randomized controlled trials & 1 & 4 \\
\hline \multirow{2}{*}{\multicolumn{3}{|c|}{$\begin{array}{l}\text { Well-conducted non-randomized comparative studies } \\
\text { Cohort studies }\end{array}$}} \\
\hline & & \\
\hline \multicolumn{3}{|l|}{ Level 3} \\
\hline \multicolumn{3}{|l|}{ Control case study } \\
\hline \multicolumn{3}{|l|}{ Level 4} \\
\hline Comparative studies with significant biases & 8 & 4 \\
\hline Retrospective studies & 15 & 19 \\
\hline \multicolumn{3}{|l|}{ Case series } \\
\hline Descriptive epidemiological studies & & 1 \\
\hline Meta-analysis of retrospective studies & 1 & 1 \\
\hline Reviews of retrospective studies & 4 & 4 \\
\hline \multicolumn{3}{|l|}{ Clinical cases } \\
\hline \multicolumn{3}{|l|}{ Books } \\
\hline Opinions / Conferences & & \\
\hline
\end{tabular}


Annex 1. Indications for laparoscopic cholecystectomy in patients with cirrhosis

\begin{tabular}{|c|c|c|c|}
\hline Author, Year & Type of study & $\begin{array}{c}\text { Number of patients } \\
\text { (896) }\end{array}$ & Indication \\
\hline Fernandes NF et al, 2000 & Single center retrospective comparative & $\mathrm{N}=48$ & $\begin{array}{l}\text { Biliary colic : } n=32 \\
\text { Cholecystitis }: n=10 \\
\text { Acute pancreatitis : } n=6\end{array}$ \\
\hline Poggio JL et al, 2000 & Single center retrospective comparative & $N=26$ & $\begin{array}{l}\text { Biliary colic : } n=22 \\
\text { Cholecystitis : } n=1 \\
\text { Acute pancreatitis : } n=3\end{array}$ \\
\hline Eason G et al, 2001 & $\begin{array}{l}\text { Single center retrospective } \\
\text { Non-comparative }\end{array}$ & $\mathrm{N}=15$ & $\begin{array}{l}\text { Biliary colic : } n=1 \\
\text { Cholecystitis : } n=10 \\
\text { Acute pancreatitis : } n=4\end{array}$ \\
\hline Leone $\mathrm{N}$ et al, 2001 & $\begin{array}{l}\text { Single center retrospective } \\
\text { Non-comparative }\end{array}$ & $\mathrm{N}=24$ & $\begin{array}{l}\text { Unspecified symptomatic } \\
\text { gallbladder stones }\end{array}$ \\
\hline Cucinotta F et al, 2003 & $\begin{array}{l}\text { Single center retrospective } \\
\text { Non-comparative }\end{array}$ & $\mathrm{N}=22$ & $\begin{array}{l}\text { Biliary colic : } n=16 \\
\text { Cholecystitis : } n=6 \\
\text { Acute pancreatitis : } n=0\end{array}$ \\
\hline Cortıs MF et al, 2005 & $\begin{array}{l}\text { Single center retrospective } \\
\text { Non-comparative }\end{array}$ & $\mathrm{N}=14$ & $\begin{array}{l}\text { Biliary colic : } n=14 \\
\text { Cholecystitis : } n=0 \\
\text { Acute pancreatitis : } n=0\end{array}$ \\
\hline Schiff J et al, 2005 & $\begin{array}{l}\text { Single center retrospective } \\
\text { Non-comparative }\end{array}$ & $\mathrm{N}=27$ & $\begin{array}{l}\text { Unspecified symptomatic } \\
\text { gallbladder stones }\end{array}$ \\
\hline Ji W et al, 2005 & Randomized controlled trial & $\mathrm{N}=38$ & $\begin{array}{l}\text { Unspecified symptomatic } \\
\text { gallbladder stones: } n=35 \\
\text { Gallbladder polyp : } n=3\end{array}$ \\
\hline da Silveira EBV et al, 2006 & Single center retrospective comparative & $\mathrm{N}=24$ & $\begin{array}{l}\text { Biliary colic : } n=14 \\
\text { Cholecystitis : } n=4 \\
\text { Acute pancreatitis : } n=4 \\
\text { Others : } n=2\end{array}$ \\
\hline Yeh CN et al, 2006 & $\begin{array}{l}\text { Single center retrospective } \\
\text { Non-comparative }\end{array}$ & $\mathrm{N}=226$ & $\begin{array}{l}\text { Biliary colic }: n=116 \\
\text { Cholecystitis }: n=76 \\
\text { Acute pancreatitis : } n=16 \\
\text { CBD stones: } n=6 \\
\text { Gallbladder polyp : } 12\end{array}$ \\
\hline Curro G et al, 2007 & $\begin{array}{l}\text { Single center retrospective } \\
\text { Non-comparative }\end{array}$ & $N=50$ & $\begin{array}{l}\text { Biliary colic : } n=36 \\
\text { Cholecystitis : } n=14 \\
\text { Acute pancreatitis : } n=0\end{array}$ \\
\hline Mancero JMP et al, 2008 & $\begin{array}{l}\text { Single center retrospective } \\
\text { Non-comparative }\end{array}$ & $\mathrm{N}=30$ & $\begin{array}{l}\text { Biliary colic : } n=0 \\
\text { Cholecystitis }: n=30 \\
\text { Acute pancreatitis : } n=0\end{array}$ \\
\hline Leandros E et al, 2008 & $\begin{array}{l}\text { Single center retrospective } \\
\text { Non-comparative }\end{array}$ & $\mathrm{N}=34$ & $\begin{array}{l}\text { Biliary colic : } n=26 \\
\text { Cholecystitis : } n=8 \\
\text { Acute pancreatitis : } n=0\end{array}$ \\
\hline El-Awadi S et al, 2009 & Randomized controlled trial & $\mathrm{N}=55$ & $\begin{array}{l}\text { Biliary colic : } n=53 \\
\text { Cholecystitis : } n=2 \\
\text { Acute pancreatitis : } n=0\end{array}$ \\
\hline Shaikh AR et al, 2009 & $\begin{array}{l}\text { Single center retrospective } \\
\text { Non-comparative }\end{array}$ & $\mathrm{N}=20$ & $\begin{array}{l}\text { Unspecified symptomatic } \\
\text { gallbladder stones }\end{array}$ \\
\hline Pavlidis TE et al, 2009 & Single center retrospective comparative & $\mathrm{N}=38$ & $\begin{array}{l}\text { Biliary colic : } n=31 \\
\text { Cholecystitis }: n=7 \\
\text { Acute pancreatitis : } n=0\end{array}$ \\
\hline Delis S et al, 2010 & $\begin{array}{l}\text { Single center retrospective } \\
\text { Non-comparative }\end{array}$ & $\mathrm{N}=220$ & $\begin{array}{l}\text { Biliary colic }: n=122 \\
\text { Cholecystitis }: n=65 \\
\text { Acute pancreatitis : } n=33\end{array}$ \\
\hline Quillin RC et al, 2013 & $\begin{array}{l}\text { Single center retrospective } \\
\text { Non-comparative }\end{array}$ & $\mathrm{N}=94$ & $\begin{array}{l}\text { Biliary colic : } n=76 \\
\text { Cholecystitis : } n=2 \\
\text { Acute pancreatitis }: n=6 \\
\text { Gallbladder polyp : } n=3 \\
\text { Gallbladder dyskinesia }: n=3 \\
\text { Others : } n=4\end{array}$ \\
\hline
\end{tabular}


indications for cholecystectomy were hepatic colic for $559(62.4 \%)$ patients, (acute or chronic) lithiasic cholecystitis for 235 (26.2\%) patients, biliary pancreatitis for 72 $(8.0 \%)$ patients and other indications for 30 (3.3\%) patients.

\section{Comparison of the open approach and the laparoscopic approach}

Between 2000 and 2015, the open and laparoscopic approach results comparison were reported in 2 prospective randomized trials meta-analyses, 4 prospective randomized trials, one studies retrospective meta-analysis and 4 retrospective studies (Annex 2).

Annex 2. Studies comparing the results of laparoscopic (LC) and open (OC) cholecystectomies in patients with cirrhosis

\begin{tabular}{|c|c|c|c|c|c|}
\hline Author, Year & Type of study & $\begin{array}{c}\mathrm{N} \\
\text { (LC vs. OC) }\end{array}$ & $\begin{array}{l}\text { Indications } \\
\text { (Total : } n=284 \text { ) }\end{array}$ & $\begin{array}{l}\text { Thild-Pugh Score } \\
\text { A/B/C (\%) } \\
\text { (Total : } n=152)\end{array}$ & $\begin{array}{l}\text { Main results } \\
\text { (laparoscopy vs. open) }\end{array}$ \\
\hline $\begin{array}{l}\text { Poggio JL et al, } \\
2000\end{array}$ & $\begin{array}{l}\text { Single center } \\
\text { retrospective comparative }\end{array}$ & 26 vs. 24 & $\begin{array}{l}\text { Biliary colic : } n=40(80 \%) \\
\text { Acute pancreatitis7 }(14 \%) \\
\text { Acute cholecystitis : } n=3(6 \%)\end{array}$ & $\begin{array}{l}A: 71 \% \\
B: 29 \% \\
C: 0 \%\end{array}$ & $\begin{array}{l}\text { Shorter surgery duration } \\
\text { Decreased blood loss } \\
\text { Decreased complication rate } \\
\text { Shorter length of stay }\end{array}$ \\
\hline $\begin{array}{l}\text { Poniachik J, et al } \\
2002\end{array}$ & $\begin{array}{l}\text { Single center retrospective } \\
\text { comparative }\end{array}$ & 35 vs. 32 & $\mathrm{NA}^{*}$ & $\begin{array}{l}A: 74 \% \\
B: 23 \% \\
C: 3 \%\end{array}$ & $\begin{array}{l}\text { Decreased complication rate } \\
\text { Shorter length of stay }\end{array}$ \\
\hline $\begin{array}{l}\text { Puggioni A et al, } \\
2003\end{array}$ & $\begin{array}{l}\text { Meta-analysis of retrospective } \\
\text { studies }\end{array}$ & $\mathrm{N} / \mathrm{A}$ & $\mathrm{N} / \mathrm{A}$ & $\mathrm{N} / \mathrm{A}$ & $\begin{array}{l}\text { Shorter surgery duration } \\
\text { Decreased blood loss } \\
\text { Similar complication rate } \\
\text { Shorter length of stay }\end{array}$ \\
\hline $\begin{array}{l}\text { da Silveira EBV et al, } \\
2006\end{array}$ & $\begin{array}{l}\text { Single center retrospective } \\
\text { comparative }\end{array}$ & 24 vs. 52 & $N / A^{* *}$ & $N / A^{*}$ & $\begin{array}{l}\text { Shorter surgery duration } \\
\text { Decreased blood loss } \\
\text { Decreased intraoperative } \\
\text { fluid requirements } \\
\text { Decreased complication rate } \\
\text { Shorter length of stay }\end{array}$ \\
\hline $\begin{array}{l}\text { Chmielecki DK et al, } \\
2012\end{array}$ & $\begin{array}{l}\text { Retrospective multicenter } \\
\text { comparative registry }\end{array}$ & 2857 vs. 383 & $\mathrm{~N} / \mathrm{A}$ & $\mathrm{N} / \mathrm{A}$ & $\begin{array}{l}\text { Decreased blood loss } \\
\text { Decreased reoperation rate } \\
\text { Decreased rate of post- } \\
\text { operative hemorrhage } \\
\text { Decreased postoperative } \\
\text { mortality rate } \\
\text { Shorter length of stay }\end{array}$ \\
\hline $\begin{array}{l}\text { Lausten SB et al, } \\
1999\end{array}$ & Randomized controlled trial & 7 vs. 7 & Biliary colic : $n=14(100 \%)$ & $A / B: 100 \%$ & $\begin{array}{l}\text { Similar surgery durations } \\
\text { Shorter length of stay } \\
\text { Similar complication rate } \\
\text { Decreased immune response }\end{array}$ \\
\hline Ji W et al, 2005 & Randomized controlled trial & 38 vs. 42 & $\begin{array}{l}\text { Biliary colic : } n=75(93.7 \%) \\
\text { Gallbladder polyp : } n=5(6.3 \%)\end{array}$ & $\begin{array}{l}A: 50 \% \\
B: 39 \% \\
C: 11 \%\end{array}$ & $\begin{array}{l}\text { Decreased blood loss } \\
\text { Decreased complication rate } \\
\text { Shorter length of stay } \\
\end{array}$ \\
\hline El-Awadi S et al, 2009 & Randomized controlled trial & 55 vs. 55 & $\begin{array}{l}\text { Biliary colic : } n=102(92.7 \%) \\
\text { Acute cholecystitis : } n=8(7.3 \%)\end{array}$ & $\begin{array}{l}A: 85 \% \\
B: 15 \% \\
C: 0 \%\end{array}$ & $\begin{array}{l}\text { Shorter surgery duration } \\
\text { Decreased blood loss } \\
\text { Decreased abdominal wall } \\
\text { complication rate } \\
\text { Shorter length of stay }\end{array}$ \\
\hline Hamad MA et al, 2010 & Randomized controlled trial & 15 vs. 15 & Acute cholecystitis : $n=30(100 \%)$ & $\begin{array}{l}\text { A : }: 67 \% \\
B: 33 \% \\
\text { C: }: 0 \%\end{array}$ & $\begin{array}{l}\text { Similar surgery durations } \\
\text { Similar blood loss } \\
\text { Similar complication rate } \\
\text { Shorter length of stay }\end{array}$ \\
\hline Laurence JM et al, 2012 & $\begin{array}{l}\text { Meta-analysis of } \\
\text { randomizedcontrolled trials }\end{array}$ & 112 vs. 108 & - & - & $\begin{array}{l}\text { Decreased complication rate } \\
\text { Decreased infectious } \\
\text { complication rate } \\
\text { Shorter length of stay }\end{array}$ \\
\hline de Goede B et al, 2014 & $\begin{array}{l}\text { Meta-analysis of randomized } \\
\text { controlled trials }\end{array}$ & $\begin{array}{l}108 \text { vs. } 112 \\
115 \text { vs. } 119 \\
77 \text { vs. } 77\end{array}$ & - & - & $\begin{array}{l}\text { Decreased complication rate } \\
\text { Shorter length of stay }\end{array}$ \\
\hline
\end{tabular}

* The indication « gallbladder stones » was not judged relevant ; ${ }^{\star \star}$ Only available for laparoscopic cholecystectomies 
Cholecystectomy indications were reported for 284 patients. Among these, $231(81.4 \%)$ were operated for hepatic colic, 41 (14.4\%) were operated on for acute cholecystitis and 12 $(4.2 \%)$ were operated on for other aetiologies [acute biliary pancreatitis $(\mathrm{n}=7)$, vesicular polyp $(\mathrm{n}=5)]$. The Child-Pugh score was specified for 145 patients having laparoscopic cholecystectomy. Of these, 118 (81.4\%), 22
(15.2\%) and 5 (3.5\%) were ranked Child-Pugh $\mathrm{A}, \mathrm{B}$ and $\mathrm{C}$, respectively.

Three retrospective studies and 2 of the prospective randomized trials showed a decrease in blood loss for the laparoscopic approach compared to laparotomy. Two retrospective studies and 1 prospective randomized trial showed a reduction in the operating time for the laparoscopic approach

Annex 3. Morbidity and mortality after laparoscopic cholecystectomies in patients with cirrhosis

\begin{tabular}{|c|c|c|c|c|c|}
\hline Author, Year & $\begin{array}{l}\text { Number of } \\
\text { patients }\end{array}$ & $\begin{array}{c}\text { Child-Pugh Score } \\
\text { A/B/C (\%) }\end{array}$ & $\begin{array}{c}\text { Complication rate } \\
\text { (\%) }\end{array}$ & Type of complication & $\begin{array}{l}\text { Mortality } \\
\text { rate }\end{array}$ \\
\hline Fernandes NF et al, 2000 & $\mathrm{~N}=48$ & $38 / 10 / 0$ & $N=5(10.4 \%)$ & $\begin{array}{l}\text { Hemorrhage }: n=2 \\
\text { Acute peritonitis }: n=1 \\
\text { Bile leakage: } n=2\end{array}$ & $0.0 \%$ \\
\hline Poggio JL et al, 2000 & $\mathrm{~N}=26$ & $22 / 4 / 0$ & $\mathrm{~N}=5(19.2 \%)$ & $\begin{array}{l}\text { Urinary infection : } n=1 \\
\text { Urinary retention }: n=3 \\
\text { Postoperative lleus }: n=1\end{array}$ & $0.0 \%$ \\
\hline Eason G et al, 2001 & $\mathrm{~N}=15$ & $15 / 0 / 0$ & $\mathrm{~N}=0(0.0 \%)$ & $\mathrm{N} / \mathrm{A}$ & $0.0 \%$ \\
\hline Leone $\mathrm{N}$ et al, 2001 & $N=24$ & $\mathrm{~N} / \mathrm{A}$ & $N=5(20.8 \%)$ & $\begin{array}{l}\text { Hemorrhage }: n=1 \\
\text { Abdominal wall hematoma: } n=3 \\
\text { Ascites }: n=1\end{array}$ & $0.0 \%$ \\
\hline Clark JR et al, 2001 & $\mathrm{~N}=25$ & $14 / 9 / 2$ & $\mathrm{~N}=13(52.0 \%)$ & $\begin{array}{l}\text { Hemorrhage }: n=2 \\
\text { Deep vein thrombosis : } n=1 \\
\text { Abdominal wall complication }: n=6 \\
\text { Abdominal collection }: n=3 \\
\text { Cardio-pulmonary complication }: n=2\end{array}$ & $\mathrm{~N}=1(4.0 \%)$ \\
\hline Tuech JJ et al, 2002 & $\mathrm{~N}=26$ & $22 / 4 / 0$ & $\mathrm{~N}=7(26.9 \%)$ & $\mathrm{N} / \mathrm{A}$ & $0.0 \%$ \\
\hline Yeh CN et al, 2006 & $N=226$ & $193 / 33 / 0$ & $N=15(6.6 \%)$ & $\begin{array}{l}\text { Ascites infection }: n=1 \\
\text { Acute peritonitis }: n=1 \\
\text { Abdominal wall hematoma: } n=2 \\
\text { Scapular pain }: n=2 \\
\text { Postoperative infection }: n=2 \\
\text { Ileus }: n=1 \\
\text { Other }: n=6\end{array}$ & $N=2(0.9 \%)$ \\
\hline Cucinotta F et al, 2003 & $N=22$ & $12 / 10 / 0$ & $\mathrm{~N}=8(36.4 \%)$ & $\begin{array}{l}\text { Hemorrhage }: n=1 \\
\text { Abdominal wall complication }: n=3 \\
\text { Abdominal collection }: n=2 \\
\text { Pulmonary infection }: n=2\end{array}$ & $0.0 \%$ \\
\hline Cortıs MF et al, 2005 & $\mathrm{~N}=14$ & $8 / 6 / 0$ & $\mathrm{~N}=3(21.4 \%)$ & $\begin{array}{l}\text { Ascites : } n=2 \\
\text { Angor : } n=1\end{array}$ & $0.0 \%$ \\
\hline Schiff J et al, 2005 & $\mathrm{~N}=27$ & $\mathrm{~N} / \mathrm{A}$ & $\mathrm{N} / \mathrm{A}$ & $\mathrm{N} / \mathrm{A}$ & $0.0 \%$ \\
\hline Ji W et al, 2005 & $\mathrm{~N}=38$ & $19 / 15 / 4$ & $\mathrm{~N}=5(13.2 \%)$ & $\begin{array}{l}\text { Ascites }: n=2 \\
\text { Hemorrhage digestive }: n=1 \\
\text { Encephalopathy }: n=1 \\
\text { Urinary infection }: n=1 \\
\text { Pulmonary infection }: n=1\end{array}$ & $0.0 \%$ \\
\hline da Silveira EBV et al, 2006 & $\mathrm{~N}=24$ & $\mathrm{~N} / \mathrm{A}$ & $\mathrm{N}=2(8.3 \%)$ & $\mathrm{N} / \mathrm{A}$ & $\mathrm{N} / \mathrm{A}$ \\
\hline Curro G et al, 2007 & $N=50$ & $32 / 18 / 0$ & $\mathrm{~N}=12(24.0 \%)$ & $\mathrm{N} / \mathrm{A}$ & $0.0 \%$ \\
\hline Mancero JMP et al, 2008 & $\mathrm{~N}=30$ & $23 / 7 / 0$ & $\mathrm{~N}=11(36.7 \%)$ & $\begin{array}{l}\text { Abdominal wall hematoma }: n=3 \\
\text { Scapular pain }: n=2 \\
\text { Diabetes decompensation }: n=1 \\
\text { Ascites }: n=3 \\
\text { Fever }: n=1 \\
\text { Subcutaneous emphysema }: n=1\end{array}$ & $0.0 \%$ \\
\hline Leandros E et al, 2008 & $\mathrm{~N}=34$ & $23 / 11 / 0$ & $N=7(20.6 \%)$ & $\begin{array}{l}\text { Hemorrhage }: n=2 \\
\text { Cardiac arrhythmia }: n=1 \\
\text { Abdominal wall complication }: n=1 \\
\text { Abdominal collection }: n=1 \\
\text { Encephalopathy }: n=2\end{array}$ & $N=1(2.9 \%)$ \\
\hline
\end{tabular}


Annex 3. Morbidity and mortality after laparoscopic cholecystectomies in patients with cirrhosis (continuation)

\begin{tabular}{|c|c|c|c|c|c|}
\hline Author, Year & $\begin{array}{c}\text { Number of } \\
\text { patients }\end{array}$ & $\begin{array}{c}\text { Child-Pugh Score } \\
\text { A/B/C (\%) }\end{array}$ & $\begin{array}{c}\text { Complication rate } \\
(\%)\end{array}$ & Type of complication & $\begin{array}{l}\text { Mortality } \\
\text { rate }\end{array}$ \\
\hline El-Awadi S et al, 2009 & $\mathrm{~N}=55$ & $47 / 8 / 0$ & $\mathrm{~N}=7(12.7 \%)$ & $\begin{array}{l}\text { Pulmonary infection }: n=3 \\
\text { Ascites }: n=2 \\
\text { Bile leakage }: n=3 \\
\text { Abdominal wall complication }: n=1 \\
\text { Abdominal wall hernia }: n=1\end{array}$ & $0.0 \%$ \\
\hline Shaikh AR et al, 2009 & $\mathrm{~N}=20$ & $12 / 8 / 0$ & $\mathrm{~N}=3(15.0 \%)$ & $\begin{array}{l}\text { Ascites : } n=2 \\
\text { Bile leakage }: n=1\end{array}$ & $0.0 \%$ \\
\hline Pavlidis TE et al, 2009 & $\mathrm{~N}=38$ & $29 / 9 / 0$ & $\mathrm{~N}=3(7.9 \%)^{*}$ & Hemorrhage : $n=3$ & $0.0 \%$ \\
\hline Delis S et al, 2010 & $N=220$ & $194 / 26 / 0$ & $\mathrm{~N}=42(19.1 \%)$ & $\begin{array}{l}\text { Hemorrhage } e^{\star *}: n=17 \\
\text { Abdominal collection }: n=14 \\
\text { Abdominal wall complication }: n=3 \\
\text { Pulmonary infection }: n=8\end{array}$ & $0.0 \%$ \\
\hline Hamad Ma et al, 2010 & $N=15$ & $\mathrm{~N} / \mathrm{A}$ & $\mathrm{N}=5(33.3 \%)$ & $\mathrm{N} / \mathrm{A}$ & $0.0 \%$ \\
\hline Lledo JB et al, 2011 & $\mathrm{~N}=43$ & $\mathrm{~N} / \mathrm{A}$ & $\mathrm{N}=5(11.6 \%)$ & $\begin{array}{l}\text { Hemorrhage }: n=1 \\
\text { Ascites }: n=3 \\
\text { Bile leakage }: n=1\end{array}$ & $0.0 \%$ \\
\hline Quillin RC et al, 2013 & $\mathrm{~N}=94$ & $63 / 20 / 2$ & $\mathrm{~N}=32(34.0 \%)$ & $\begin{array}{l}\text { Infection }: n=15 \\
\text { Ascites }: n=6 \\
\text { Encephalopathy }: n=2 \\
\text { MOF }: n=3 \\
\text { Digestive hemorrhage }: n=1 \\
\text { lleus }: n=1 \\
\text { Abdominal wall complication }: n=2 \\
\text { Portal vein thrombosis : } n=1 \\
\text { Acute pancreatitis }: n=1 \\
\text { Anemia }: n=3 \\
\text { Deep vein thrombosis }: n=1 \\
\text { Diarrhea }: n=2 \\
\text { Dehydration }: n=1\end{array}$ & $\mathrm{~N}=4(4.3 \%)$ \\
\hline
\end{tabular}

* « Severe » complications ; ** Intra-operative hemorrhage

compared to laparotomy. Three retrospective studies and 1 prospective randomized trial showed a decrease in the rate of overall complications for the laparoscopic approach compared to laparotomy. Finally, the 4 retrospective studies and the 4 prospective randomized trials demonstrated a reduction in the length of stay for the laparoscopic approach compared to laparotomy. The meta-analysis of retrospective studies showed a reduction in operating time, blood loss and length of stay for the laparoscopic approach compared to laparotomy, but reported similar rates of complications between the two approaches. The two metaanalysis of prospective randomized trials reported a reduction in the complication rate and length of stay for the laparoscopic approach compared to laparotomy.

In total, due to better results in terms of blood loss, postoperative complications and length of stay, the laparoscopic approach should be favored in Child A and B classified cirrhotic patients, operated for "hepatic colic" (rank 1level of evidence). For Child A and $\mathrm{B}$ cirrhotic patients requiring cholecystectomy secondary to acute lithiasic cholecystitis, the laparoscopic approach is associated with length of stay reduction and also seems to be preferred (rank 2 level of evidence).

\section{Conversion to laparotomy during laparoscopic cholecystectomy in cirrhotic patients}

Twenty-six articles published between 2000 and 2015, including 23 retrospective studies and 3 prospective randomized trials, reported conversion rates for a total of 4,029 cirrhotic patients operated on for laparoscopic cholecystectomy (Annex 4). Conversion to laparotomy was performed for $494(12.3 \%)$ patients with rates varying from $0 \%$ to $20.8 \%$. Conversions details were reported in 12 studies, but only 
Annex 4. Causes and rate of conversion to an open approach in patients with cirrhosis undergoing laparoscopic cholecystectomy

\begin{tabular}{|c|c|c|c|c|}
\hline Author, Year & Type of study & $\begin{array}{c}\text { Number of } \\
\text { patients }\end{array}$ & $\begin{array}{c}\text { Conversion } \\
(\%)\end{array}$ & $\begin{array}{l}\text { Cause for conversion } \\
\text { (Total : } n=46 \text { ) }\end{array}$ \\
\hline Fernandes NF et al, 2000 & $\begin{array}{l}\text { Single center retrospective } \\
\text { Non-comparative }\end{array}$ & $\mathrm{N}=48$ & $\mathrm{~N}=4(8.3 \%)$ & $\begin{array}{l}\text { Adhesions: } n=2 \\
\text { Intraoperative discovery of liver mass: } n=1 \\
\text { Cystic artery bleeding }: n=1\end{array}$ \\
\hline Poggio JL et al, 2000 & $\begin{array}{l}\text { Single center retrospective } \\
\text { comparative }\end{array}$ & $N=26$ & $\mathrm{~N}=3(11.5 \%)$ & $\begin{array}{l}\text { Gallbladder bed hemorrhage: } n=2 \\
\text { Poor visualization of surrounding structures: } n=1\end{array}$ \\
\hline Morino $\mathrm{M}$ et al, 2000 & $\begin{array}{l}\text { Single center retrospective } \\
\text { Non-comparative }\end{array}$ & $N=33$ & $\mathrm{~N}=2(6.1 \%)$ & - \\
\hline Urban L et al, 2001 & $\begin{array}{l}\text { Single center retrospective } \\
\text { Non-comparative }\end{array}$ & $N=15$ & $\mathrm{~N}=3(20.0 \%)$ & $\begin{array}{l}\text { Adhesions : } n=1 \\
\text { Significant inflammation }: n=1 \\
\text { Intraoperative discovery of metastatic pancreatic } \\
\text { adenocarcinoma }: n=1\end{array}$ \\
\hline Leone $\mathrm{N}$ et al, 2001 & $\begin{array}{l}\text { Single center retrospective } \\
\text { Non-comparative }\end{array}$ & $N=24$ & $\mathrm{~N}=1(4.2 \%)$ & $\mathrm{N} / \mathrm{A}$ \\
\hline Tuech JJ et al, 2002 & $\begin{array}{l}\text { Single center retrospective } \\
\text { Non-comparative }\end{array}$ & $N=26$ & $\mathrm{~N}=0(0.0 \%)$ & - \\
\hline Yeh CN et al, 2002 & $\begin{array}{l}\text { Single center retrospective } \\
\text { Non-comparative }\end{array}$ & $N=226$ & $\mathrm{~N}=10(4.4 \%)$ & $\mathrm{N} / \mathrm{A}$ \\
\hline Clark JR et al, 2003 & $\begin{array}{l}\text { Single center retrospective } \\
\text { Non-comparative }\end{array}$ & $\mathrm{N}=25$ & $\mathrm{~N}=0(0.0 \%)$ & - \\
\hline Cucinotta F et al, 2003 & $\begin{array}{l}\text { Single center retrospective } \\
\text { Non-comparative }\end{array}$ & $N=22$ & $\mathrm{~N}=2(9.1 \%)$ & Adhesions : $n=2$ \\
\hline Cortis MF et al, 2005 & $\begin{array}{l}\text { Single center retrospective } \\
\text { Non-comparative }\end{array}$ & $N=14$ & $\mathrm{~N}=0(0.0 \%)$ & - \\
\hline Schiff J et al, 2005 & $\begin{array}{l}\text { Single center retrospective } \\
\text { Non-comparative }\end{array}$ & $N=27$ & $\mathrm{~N}=3(11.1 \%)$ & $\begin{array}{l}\text { Unexperienced surgeon : } n=2 \\
\text { Difficult exposure }: n=1\end{array}$ \\
\hline Ji W et al, 2005 & Randomized controlled trial & $\mathrm{N}=38$ & $\mathrm{~N}=2(5.3 \%)$ & $\begin{array}{l}\text { Gallbladder bed hemorrhage: } n=1 \\
\text { Adhesions : } n=1\end{array}$ \\
\hline da Silveira EBV et al, 2006 & $\begin{array}{l}\text { Single center retrospective } \\
\text { Comparative }\end{array}$ & $N=24$ & $N=5(20.8 \%)$ & $\mathrm{N} / \mathrm{A}$ \\
\hline Curro G et al, 2007 & $\begin{array}{l}\text { Single center retrospective } \\
\text { Non-comparative }\end{array}$ & $N=50$ & $N=2(4 \%)$ & Significant inflammation : $n=2$ \\
\hline Mancero JMP et al, 2008 & $\begin{array}{l}\text { Single center retrospective } \\
\text { Non-comparative }\end{array}$ & $\mathrm{N}=30$ & $\mathrm{~N}=0(0.0 \%)$ & - \\
\hline Leandros E et al, 2008 & $\begin{array}{l}\text { Single center retrospective } \\
\text { Non-comparative }\end{array}$ & $N=34$ & $\mathrm{~N}=3(8.8 \%)$ & $\begin{array}{l}\text { Gallbladder bed hemorrhage: } n=2 \\
\text { Poor visualization of surrounding structures: } n=1\end{array}$ \\
\hline El-Awadi S et al, 2009 & Randomized controlled trial & $\mathrm{N}=55$ & $\mathrm{~N}=4(7.3 \%)$ & $\begin{array}{l}\text { Gallbladder bed hemorrhage: } n=2 \\
\text { Poor visualization of surrounding structures: } n=2\end{array}$ \\
\hline Shaikh AR et al, 2009 & $\begin{array}{l}\text { Single center retrospective } \\
\text { Non-comparative }\end{array}$ & $N=20$ & $\mathrm{~N}=2(10.0 \%)$ & Gallbladder bed hemorrhage: $n=2$ \\
\hline Pavlidis TE et al, 2009 & $\begin{array}{l}\text { Single center retrospective } \\
\text { Non-comparative }\end{array}$ & $\mathrm{N}=38$ & $\mathrm{~N}=6(15.8 \%)$ & $\begin{array}{l}\text { Poor visualization of surrounding structures: } n=3 \\
\text { Gallbladder bed hemorrhage: } n=3\end{array}$ \\
\hline Delis S et al, 2010 & $\begin{array}{l}\text { Single center retrospective } \\
\text { Non-comparative }\end{array}$ & $N=220$ & $\mathrm{~N}=12(5.5 \%)$ & $\begin{array}{l}\text { Gallbladder bed hemorrhage: } n=7 \\
\text { Poor visualization of surrounding structures: } n=5\end{array}$ \\
\hline Telem DA et al, 2010 & $\begin{array}{l}\text { Single center retrospective } \\
\text { Non-comparative }\end{array}$ & $N=26$ & $\mathrm{~N}=2(7,7 \%)$ & - \\
\hline Hamad Ma et al, 2010 & Randomized controlled trial & $\mathrm{N}=15$ & $\mathrm{~N}=1(6.7 \%)$ & - \\
\hline Lledo JB et al, 2011 & $\begin{array}{l}\text { Single center retrospective } \\
\text { Non-comparative }\end{array}$ & $N=43$ & $N=5(11.6 \%)$ & $\mathrm{N} / \mathrm{A}$ \\
\hline Chmielecki DK et al, 2012 & $\begin{array}{l}\text { Retrospective comparative } \\
\text { multicenter registry }\end{array}$ & $N=2857$ & $\mathrm{~N}=412(14.4 \%)$ & $\mathrm{N} / \mathrm{A}$ \\
\hline Quillin RC et al, 2013 & $\begin{array}{l}\text { Single center retrospective } \\
\text { Non-comparative }\end{array}$ & $\mathrm{N}=94$ & $\mathrm{~N}=10(10.6 \%)$ & $\mathrm{N} / \mathrm{A}$ \\
\hline
\end{tabular}


involved 46 patients. The most frequently mentioned causes were the existence of a vesicular bed haemorrhage for 19 (41.3\%) patients, structures insufficient visualization for $12(26.1 \%)$ patients, the existence of adhesions for $6(13.0 \%)$ patients, inflammation too great to allow safe laparoscopic surgery for 3 patients (6.5\%) and another cause for 6 $(13.0 \%)$ patients.

Only one study looked at the analysis of conversion risk factors (41). In this noncomparative unicentric retrospective study analysing 94 cirrhotic patients laparoscopically operated, $10(10.6 \%)$ patients had required conversion to laparotomy, and the reasons were not specified. In univariate analysis, a decrease in preoperative albumin levels, an increase in the preoperative MELD score, and an increase in intraoperative blood loss were significantly associated with conversion to laparotomy. In multivariate analysis, only an increase in blood loss was significantly associated with conversion to laparotomy.

Only one study looked at the postoperative course of patients who required conversion (46). In this comparative multicentre register, 383 cirrhotic patients operated by laparotomy were compared to 2,857 cirrhotic patients laparoscopically operated. Amongst the latter, the intervention was carried out laparoscopically for $2445(85.6 \%)$ of them and $412(14.4 \%)$ had required conversion, and the reasons were not specified. Compared to laparotomy operated patients, conversion requiring patients had significantly lower postoperative infection rates $(3.5 \%$ vs. $0.2 \%, \mathrm{p}<0.001)$, postoperative haemorrhage $(6.6 \%$ vs. $9.4 \%, \mathrm{p}=$ $0.001)$ and postoperative mortality ( $8.3 \%$ vs. $1.3 \%, \mathrm{p}<0.001)$. The transfusion rates were not significantly different between these two groups $(19.2 \%$ vs. $14.4 \%, p=0.100)$. Finally, the revision surgery rates were significantly higher for patients requiring conversion compared to laparotomy operated patients $(2.5 \%$ vs. $1.5 \%, \mathrm{p}=0.002)$. Conversion requiring patients were significantly more often transfused $(14.4 \%$ vs. $6.2 \%, \mathrm{p}<0.001)$, suffered more frequent complications by postoperative haemorrhage $(3.0 \%$ vs. $9.3 \%$, $\mathrm{p}<0.001)$ and required more frequent another surgery ( $0.8 \%$ vs. $2.5 \%, \mathrm{p}<0.001)$. The operative mortality rates were similar between these two groups $(1.4 \%$ vs. $1.3 \%, p=0.676)$. Finally, the postoperative infection rate was significantly higher for patients who did not require conversion compared to patients who required conversion $(0.7 \%$ vs. $0.2 \%, p<0.001)$.

In total, an open route conversion in a laparoscopically operated cirrhotic patient does not seem to be detrimental to the postoperative results compared to the open approach (rank 4 level of evidence). The literature does not make it possible to determine a subgroup of patients at high risk of conversion for whom it is preferable to perform a laparotomy immediately. Apart from pneumoperitoneum contraindication patients, it is however justified to immediately consider an open cholecystectomy in cirrhotic patients with a heavy history of abdominal surgery, in particular above mezocolium area (rank 4 level of evidence).

Technical peculiarities inherent in the cirrhotic patient and partial cholecystectomy

The cholecystectomy inherent technical difficulties in cirrhotic patients are multiple and can be grouped as follows: 1) risk of a periumbilical collateral wound during optical trocar placement; 2) haemorrhage risk vascular adhesions; 3) liver difficult traction with Calot triangle difficult exposure; 4) vesicular pedicle risky approach in the context of portal hypertension; and 5) vesicular bed haemorrhagic dissection (47). These difficulties are even more exacerbated in acute or chronic cholecystitis operated patients.

In these circumstances, Palanivelu et al. formalized certain technical aspects of laparoscopic cholecystectomy for cirrhotic patients, such as the placement of an optical trocar in the sub-umbilical position; the use of Ultracision for adhesions dissection; the placement of an additional $5 \mathrm{~mm}$ trocar to the right of the usual epigastric trocar to allow the use of a liver retractor; performing a partial cholecystectomy to avoid significant bleeding during gallbladder bed dissection or to limit 
the risk of biliary injury during cystic pedicle dissection, and, finally, the systematic placement of an abdominal drain at the end of the procedure. Thus, in their non-comparative retrospective series of 265 patients, the authors report that the use of such technical devices had made it possible to obtain $1.5 \%$ transfusion rates, a zero postoperative mortality rate and 4 days hospital stays. In addition, although $52.8 \%$ of the patients presented a bilious discharge postoperatively, this discharge had dried up spontaneously in less than 7 days for $98.6 \%$ of them. Similarly, a recent meta-analysis looking at difficult situations partial cholecystectomy results of $72.9 \%$ of laparoscopic partial cholecystectomies and $18.2 \%$ of cirrhotic patients with portal hypertension reported results similar to those observed during standard circumstances performed cholecystectomies (48).

In total, if as in laparotomy most authors perform laparoscopic cholecystectomy in the usual way, performing a partial cholecystectomy seems to be an interesting alternative in difficult situations, especially in cirrhotic patients with stigmata of portal hypertension or context of cholecystitis (rank 4 level of evidence).

\section{Draining the abdominal cavity}

While the installation of a drain in contact with the vesicular bed following an uncomplicated laparoscopic cholecystectomy was the subject of a meta-analysis (49) not showing any benefit of drainage in this context, the setting in place of an abdominal drain in cirrhotic patients remains controversial. On the one hand, the postoperative haemorrhagic complications increased risk in this difficult dissection context could encourage an increased monitoring during the procedure. On the other hand, the possibility of ascites fluid ascending infection risk would discourage the systematic leave of a drain.

Amongst all the articles dealing with laparoscopic cholecystectomy for cirrhotic patients, only 4 specified whether an abdominal drain had been left in place after surgery, including two prospective randomized trials and two retrospective studies (Appendix 5). The indications for cholecystectomy were

Annex 5. Drainage of the abdominal cavity in patients with cirrhosis undergoing laparoscopic cholecystectomy

\begin{tabular}{|c|c|c|c|c|c|}
\hline Author, Year & $\begin{array}{l}\text { Number of } \\
\text { patients }\end{array}$ & $\begin{array}{c}\text { Child-Pugh Score } \\
\text { A/B/C (\%) }\end{array}$ & $\begin{array}{l}\text { Drainage of the } \\
\text { abdominal cavity }\end{array}$ & Type of complication & $\begin{array}{l}\text { Mortality } \\
\text { rate }\end{array}$ \\
\hline Fernandes NF et al, 2000 & $\mathrm{~N}=48$ & $38 / 10 / 0$ & $\mathrm{~N} / \mathrm{A}$ & $\begin{array}{l}\text { Hemorrhage }: n=2 \\
\text { Acute peritonitis : } n=1 \\
\text { Bile leakage: } n=2\end{array}$ & $0.0 \%$ \\
\hline Poggio JL et al, 2000 & $\mathrm{~N}=26$ & $22 / 4 / 0$ & $\mathrm{~N} / \mathrm{A}$ & $\begin{array}{l}\text { Urinary infection : } n=1 \\
\text { Urinary retention }: n=3 \\
\text { Postoperative ileus }: n=1\end{array}$ & $0.0 \%$ \\
\hline Eason $\mathrm{G}$ et al, 2001 & $\mathrm{~N}=15$ & $15 / 0 / 0$ & $\mathrm{~N} / \mathrm{A}$ & $\mathrm{N} / \mathrm{A}$ & $0.0 \%$ \\
\hline Leone $\mathrm{N}$ et al, 2001 & $\mathrm{~N}=24$ & $\mathrm{~N} / \mathrm{A}$ & $\mathrm{N} / \mathrm{A}$ & $\begin{array}{l}\text { Hemorrhage : } n=1 \\
\text { Abdominal wall hematoma: } n=3 \\
\text { Ascites }: n=1\end{array}$ & $0.0 \%$ \\
\hline Yeh CN et al, 2006 & $\mathrm{~N}=226$ & $193 / 33 / 0$ & $\mathrm{~N} / \mathrm{A}$ & $\begin{array}{l}\text { Ascites infection : } n=1 \\
\text { Acute peritonitis : } n=1 \\
\text { Abdominal wall hematoma: } n=2 \\
\text { Scapular pain }: n=2 \\
\text { Postoperative infection }: n=2 \\
\text { lleus }: n=1 \\
\text { Other }: n=6\end{array}$ & $\mathrm{~N}=2(0.9 \%)$ \\
\hline Cucinotta F et al, 2003 & $\mathrm{~N}=22$ & $12 / 10 / 0$ & $\mathrm{~N} / \mathrm{A}$ & $\begin{array}{l}\text { Hemorrhage }: n=1 \\
\text { Abdominal wall complication }: n=3 \\
\text { Abdominal collection }: n=2 \\
\text { Pulmonary infection }: n=2\end{array}$ & $0.0 \%$ \\
\hline Cortis MF et al, 2005 & $\mathrm{~N}=14$ & $8 / 6 / 0$ & $\mathrm{~N} / \mathrm{A}$ & $\begin{array}{l}\text { Ascites : } \mathrm{n}=2 \\
\text { Angor }: \mathrm{n}=1\end{array}$ & $0.0 \%$ \\
\hline
\end{tabular}


Annex 5. Drainage of the abdominal cavity in patients with cirrhosis undergoing laparoscopic cholecystectomy (continuation)

\begin{tabular}{|c|c|c|c|c|c|}
\hline Author, Year & $\begin{array}{l}\text { Number of } \\
\text { patients }\end{array}$ & $\begin{array}{c}\text { Child-Pugh Score } \\
\text { A/B/C (\%) }\end{array}$ & $\begin{array}{l}\text { Drainage of the } \\
\text { abdominal cavity }\end{array}$ & Type of complication & $\begin{array}{l}\text { Mortality } \\
\text { rate }\end{array}$ \\
\hline Schiff J et al, 2005 & $\mathrm{~N}=27$ & $\mathrm{~N} / \mathrm{A}$ & $\mathrm{N} / \mathrm{A}$ & $\mathrm{N} / \mathrm{A}$ & $0.0 \%$ \\
\hline Ji W et al, 2005 & $\mathrm{~N}=38$ & $19 / 15 / 4$ & $\begin{array}{c}\text { Routine } \\
\mathrm{N}=38(100 \%)\end{array}$ & $\begin{array}{l}\text { Ascites : } n=2 \\
\text { Hemorrhage digestive }: n=1 \\
\text { Encephalopathy }: n=1 \\
\text { Urinary infection }: n=1 \\
\text { Pulmonary infection }: n=1\end{array}$ & $0.0 \%$ \\
\hline da Silveira EBV et al, 2006 & $\mathrm{~N}=24$ & N/A & N/A & $\mathrm{N} / \mathrm{A}$ & $\mathrm{N} / \mathrm{A}$ \\
\hline Curro $\mathrm{G}$ et al, 2007 & $\mathrm{~N}=50$ & $32 / 18 / 0$ & $\mathrm{~N} / \mathrm{A}$ & $\mathrm{N} / \mathrm{A}$ & $0.0 \%$ \\
\hline Mancero JMP et al, 2008 & $\mathrm{~N}=30$ & $23 / 7 / 0$ & $\begin{array}{c}\text { Elective } \\
\mathrm{N}=2(6.7 \%)\end{array}$ & $\begin{array}{l}\text { Abdominal wall hematoma }: n=3 \\
\text { Scapular pain }: n=2 \\
\text { Diabetes decompensation }: n=1 \\
\text { Ascites }: n=3 \\
\text { Fever }: n=1 \\
\text { Subcutaneous emphysema }: n=1\end{array}$ & $0.0 \%$ \\
\hline Leandros E et al, 2008 & $\mathrm{~N}=34$ & $23 / 11 / 0$ & N/A but avoided & $\begin{array}{l}\text { Hemorrhage : } n=2 \\
\text { Cardiac arrhythmia }: n=1 \\
\text { Abdominal wall complication }: n=1 \\
\text { Abdominal collection }: n=1 \\
\text { Encephalopathy }: n=2\end{array}$ & $\mathrm{~N}=1(2.9 \%)$ \\
\hline El-Awadi S et al, 2009 & $N=55$ & $47 / 8 / 0$ & $\mathrm{~N}=55(100 \%)$ & $\begin{array}{l}\text { Pulmonary infection : } n=3 \\
\text { Ascites }: n=2 \\
\text { Bile leakage }: n=3 \\
\text { Abdominal wall complication }: n=1 \\
\text { Abdominal wall hernia }: n=1\end{array}$ & $0.0 \%$ \\
\hline Shaikh AR et al, 2009 & $\mathrm{~N}=20$ & $12 / 8 / 0$ & $\mathrm{~N}=20(100 \%)$ & $\begin{array}{l}\text { Ascites : } n=2 \\
\text { Bile leakage }: n=1\end{array}$ & $0.0 \%$ \\
\hline Pavlidis TE et al, 2009 & $\mathrm{~N}=38$ & $29 / 9 / 0$ & N/A & Hemorrhage : $n=3$ & $0.0 \%$ \\
\hline Delis S et al, 2010 & $\mathrm{~N}=220$ & $194 / 26 / 0$ & $\begin{array}{l}\text { N/A but elective in case } \\
\text { of difficult dissection }\end{array}$ & $\begin{array}{l}\text { Hemorrhage }^{\star *}: n=17 \\
\text { Abdominal collection }: n=14 \\
\text { Abdominal wall complication }: n=3 \\
\text { Pulmonary infection }: n=8\end{array}$ & $0.0 \%$ \\
\hline Quillin RC et al, 2013 & $N=94$ & $63 / 20 / 2$ & N/A & $\begin{array}{l}\text { Infection }: n=15 \\
\text { Ascites }: n=6 \\
\text { Encephalopathy }: n=2 \\
\text { MOF }: n=3 \\
\text { Hemorrhage digestive }: n=1 \\
\text { lleus }: n=1 \\
\text { Abdominal wall complication }: n=2 \\
\text { Portal vein thrombosis }: n=1 \\
\text { Acute pancreatitis : } n=1 \\
\text { Anemia }: n=3 \\
\text { Deep vein thrombosis : } n=1 \\
\text { Diarrhea }: n=2 \\
\text { Dehydration }: n=1\end{array}$ & $\mathrm{~N}=4(4.3 \%)$ \\
\hline
\end{tabular}

* « Severe » complications ; ** Intra-operative hemorrhage

specified in 2 of these studies for a total of 85 patients and were dominated by hepatic colic (62.4\%), followed by cholecystitis (37.6\%). Two prospective randomized trials and a retrospective study reported systematic use of a drain in contact with the gallbladder bed, and one article reported elective use of an abdominal drain due to postoperative haemorrhage increased risk (37). A retrospective study including 30 patients reported case-by-case drainage with a $6.7 \%$ rate (36). In addition, a retrospective study reported a minimal drainage attitude without specifying the number of patients drained (39). Finally, the only study reporting the subtotal cholecystectomy results in cirrhotic patients mentioned the installation of a systematic drain during the final intervention. None of the main 
objective of the studies was to analyse the abdominal drainage relevance and none included the drainage parameter as postoperative complications risk factor.

The abdominal drainage relevance for cirrhotic patients undergoing hepatectomy has been analysed in at least two prospective randomized trials $(50,51)$ showing that the systematic placement of an abdominal drain during intervention's final stage was a postoperative complications risk factor. Thus, the hepatic surgery currently adopted attitude is to assess drainage placement for each individual case in patients who have required complex resection, at high risk of biliary fistula or haemorrhage.

In total, the literature does not recommend the establishment or absence of an abdominal drain after laparoscopic cholecystectomy for cirrhotic patients. A reasonable attitude nevertheless seems to leave a drain in contact with the vesicular bed on a case-by-case basis, in particular in difficult or haemorrhagic surgery context and in patients requiring a partial cholecystectomy (rank 4 level of evidence).

\section{Lithiasis of the main bile duct in cirrhotic patients}

A meta-analysis recently highlighted the superiority of open choledocotomy for main bile duct stones extraction in non-cirrhotic patients compared to the endoscopic approach in terms of lithiasic clearance rate without significant increase in postoperative morbidity and mortality while the laparoscopic approach brought similar results to the endoscopic approach (52).

At the present time, no study has looked at the comparison of these different approaches for cirrhotic patients. Only one study looked at the endoscopic retrograde cholangiopancreatography (ERCP) management subsequent to main bile duct obstructions in cirrhotic patients (53). This study included 538 procedures performed in cirrhotic patients, of which 35 $(6.5 \%)$ for main bile duct lithiasis. If the authors reported satisfactory overall results, the lithiasic pathology ERCP results were not detailed. One study looked at the laparoscopic approach results in main bile duct lithiasis management of cirrhotic patients. In this Chinese retrospective study including 346 patients, counting 132 (38.2\%) sick Child A and $214(61.8 \%)$ sick Child B, the authors reported a $5.7 \%$ conversion rate, a $9.6 \%$ overall complication rate and a zero mortality rate (54). Although Child B patients had greater blood loss than Child A patients ( 85 vs. $35 \mathrm{~mL}, \mathrm{p}$ $<0.01$ ), both groups had similar operating times (2.1 vs. 1.9 hours, $\mathrm{P}=0.07$ ), conversion rates $(5.3 \%$ vs. $6.1 \% ; \mathrm{p}=0.77)$, complication rates $(10.6 \%$ vs. $8.8 \% ; p=0.60)$, residual lithiasis rates $(8.3 \%$ vs. $7.1 \%, p=0.65)$, and length of stay ( 4.2 vs. 4.0 days, $p=0.60)$. These results should nevertheless be interpreted with caution due to authors dubious probity, for they had to subsequently withdraw their publication (the reason being dual publication) (55).

Overall, view the literature data absence, it is impossible to recommend a certain surgical approach in the cirrhotic patient's main bile duct lithiasis management, which will therefore be at best treated by interventional endoscopy (rank 4 level of evidence).

\section{Non-lithiasic biliary pathology of cirrhotic patients}

The therapeutic possibilities of cirrhotic patients non-lithiasic biliary pathology depend on this pathology nature (tumour, inflammatory, mechanical, viral, iatrogenic etc.) and include drug treatments use, dilation and stents placement by endoscopic or percutaneous route and other various surgical procedures.

Only one study looked at endoscopic retrograde cholangiopancretaography (ERCP) management of main bile duct obstructions in cirrhotic patients (53). In this retrospective study, 538 procedures were performed in cirrhotic patients, of which $229(42.6 \%)$ Child A, 229 (42.6\%) Child B and 80 (14.8\%) Child C. The indications for ERCP were main bile duct stenosis for $379(70.4 \%)$ patients mainly in context of CSP, main bile duct lithiasis for 35 
(6.5\%) patients, recurrent pancreatitis for 22 (4.1\%) patients, and another cause for 102 (19.0\%) patients. The post-procedural complication rate was $9.1 \%$, of which $4.6 \%$ was acute pancreatitis, $2.8 \%$ cholangitis, $1.1 \%$ haemorrhage, $0.9 \%$ pneumonia, $0.4 \%$ duodenal perforation, $0.2 \%$ acute cholecystitis, $0.2 \%$ biliary fistula. The post-procedure mortality rate was $0.2 \%$. The complication rate was significantly associated with the severity of the underlying liver pathology (Child A: $11.4 \%$, Child B: $11.3 \%$ and Child C: $6.1 \%, p=0.048$ ) and the achievement of " an ERCP for a pathology other than a CSP $(19.8 \%$ vs. $4.5 \%, p<0.001)$. Long-term follow-up of patients was not evaluated.

Only one study was concerned with the non-lithiasic biliary pathology surgical management of cirrhotic patients spanning the 2000-2015 study period and dealt with the main bile duct stenosis management in the context of primary sclerosing cholangitis (CSP) (56). In this study including 126 patients with main bile duct stenosis or dominant stenosis linked to CSP, $61.1 \%$ had had resection of the main bile duct with hepaticojejunal anastomosis on biliary convergence associated with bilateral intrahepatic silicone stents placement and $39.9 \%$ had had a liver transplant. Among the 77 patients with main bile duct resection, the vast majority of patients were operated due to symptomatology persistence despite biliary drainage by endoscopic or percutaneous route in $61.0 \%$ and $67.5 \%$ of cases, respectively. Only $9(11.6 \%)$ were cirrhotic compared to $39(79.6 \%)$ transplant patients. While the overall complication and mortality rates after main bile duct resection were $38.5 \%$ and respectively $3.9 \%$, they were not cirrhotic patients-specific. Nevertheless, the authors reported that 2 $(22.2 \%)$ cirrhotic patients had liver failure requiring liver transplantation one year after the procedure. The survival rates at 3.5 and 10 years after main bile duct resection were $85.4 \%, 76.4 \%$, and respectively, $52.7 \%$, and were significantly lower for cirrhotic patients compared to non-cirrhotic patients $(60.0 \%$, $36.0 \%, 12.0 \%$ vs. $89.6 \%, 83.3 \%$ and $60.2 \%$, $\mathrm{p}<0.001$ ). Thus, the authors concluded that for cirrhotic patients with main bile duct stenosis linked to a CSP endoscopic treatment resistant, the main bile duct resection place was only very limited and that these patients should have liver transplantation priority even if liver function is preserved.

As for the rest of the non-lithiasis main bile duct pathology, no study has specifically looked at cirrhotic patients.

Overall, in the context of cirrhosis patients, main bile duct stenoses linked to primary sclerosing cholangitis must be treated with ERCP as a first intention. In the event of failure, liver transplantation should be favoured even in the presence of preserved liver function (rank 4 level of evidence). Regarding the rest of main bile duct nonlithiasic pathology, no study has specifically looked at cirrhotic patients, and it is therefore not possible to establish literature-based recommendations (rank $\infty$ level of evidence).

\section{Results of Surgery: Morbidity and Mortality}

Twenty-two articles published between 2000 and 2015 reported complication rates for cirrhotic patients with laparoscopic cholecystectomy (Appendix 3). Among these, there were 3 prospective randomized trials and 19 retrospective studies for a total of 1107 patients included. A total of 195 (17.6\%) patients presented with at least one postoperative complication.

Sixteen articles, including 2 prospective randomized trials and 14 retrospective studies, detailed the type of postoperative complications for a total of 947 analysable patients. The most frequently reported complications were: 1 ) postoperative infection for $36(3.8 \%)$ patients including 14 (1.5\%) pneumopathies, 2) parietal complication for 24 $(2.5 \%)$ patients, 3) postoperative ascites for 22 (2.3\%) patients, 4) abdominal collection for 20 $(2.1 \%)$ patients, 5$)$ postoperative haemorrhage for $12(1.3 \%)$ patients, and 6) biliary fistula for $7(0.7 \%)$ patients.

Only two articles looked at postoperative complications risk factors for cirrhotic patients with laparoscopic cholecystectomy 
$(39,41)$. The retrospective study by Delis et al. included 220 cirrhotic patients (Child A: $88.2 \%$ and Child B: $11.8 \%$ ), of which $55.5 \%$ were operated for hepatic colic, $29.5 \%$ for acute cholecystitis and $15.0 \%$ following an episode of acute biliary pancreatitis. The postoperative complication rate was $19.1 \%$ and was significantly increased for patients with a MELD score $>13(45.8 \%$ vs. $11.6 \%, p=0.045)$ but not by the Child (Child) score A: $18.5 \%$ vs. $23.0 \%$ Child B, $p=0.19$ ). The retrospective study by Quillin et al. included 94 cirrhotic patients (Child A: 64.9\%, Child B: 21.3\% and Child C: $2.1 \%), 81 \%$ of whom were operated for hepatic colic, 6\% following an episode acute biliary pancreatitis, $3 \%$ for vesicular polyp, $3 \%$ for vesicular dyskinesia, $2 \%$ for acute cholecystitis and $4 \%$ for other causes. The complication rate was $34 \%$ and was significantly associated with the male sex ( $66 \%$ vs. $42 \%$, p < 0.03$)$, with a low preoperative albumin level ( $3.5 \mathrm{~g} / \mathrm{dL}$ vs. $3.9 \mathrm{~g} / \mathrm{dL}, \mathrm{p}<0.01$ ), an increase in preoperative INR ( 1.2 vs. $1.1, \mathrm{p}<0.05$ ), an MELD score> 10 (60\% vs. $31 \%$, p <0.02), an increased Child score ( 6 vs. $5, \mathrm{p}<0.01$ ), and a higher number of blood cells transfused intraoperatively (0.4 CG vs. $0.03 \mathrm{CG}, \mathrm{p}<0.04$ ).

Sixteen articles concomitantly specified the details of Child's classification and the postoperative mortality rate (Appendix 3). Hence, among the 970 patients who could be analysed, there were $766(79.0 \%)$ Child A patients, $196(20.2 \%)$ Child B patients and 8 $(0.8 \%)$ Child $\mathrm{C}$ patients. In total, $8(0.7 \%)$ patients died following the intervention, of which $3(37.5 \%)$ patients were classified Child A, $2(25.0 \%)$ were classified Child B and 3 (37.5\%) were classified Child C. The mortality rates for patients classified Child $\mathrm{A}, \mathrm{B}$ and $\mathrm{C}$ were thus $0.2 \%, 1.0 \%$ and respectively $37.5 \%$.

Only one retrospective study looked at laparoscopic cholecystectomy mortality risk factors in cirrhotic patients (41). In this study, the postoperative mortality rate was $4.3 \%$ and significantly associated with an increase in the INR preoperative ( 1.4 vs. $1.1, \mathrm{p}<0.02)$, an increased Child score ( 8 vs. $6, \mathrm{p}<0.03$ ), a higher number of blood cells transfused intraoperatively (2.3 CG vs. 0.07 CG, p <0.04) and a higher number of intraoperatively transfused platelets ( 0.8 vs. $0.2, \mathrm{p}<0.02$ ).

Finally, a retrospective unicentric study including 78 cirrhotic patients operated via various procedures analysed the postoperative morbidity and mortality risk factors (57). In this study, $35(44.9 \%)$ patients were operated on for an umbilical hernia, $17(21,8 \%)$ of a colectomy and $26(33.3 \%)$ patients had a laparoscopic cholecystectomy. Among these, 20 were classified Child A, 5 were classified Child $\mathrm{B}$ and 1 was classified Child C. The authors reported complication rates of $23.1 \%$ including $15 \%$ for patients with Child A, $40 \%$ for patients with Child B and 100\% for the only Child C patient. One patient (3.8\%) classified Child B but with a MELD > 15 died following the intervention. The risk factors for postoperative complication in multivariate analysis for all 78 procedures performed were: an ASA score $>3$, an emergency surgery, blood loss > $150 \mathrm{~mL}$, the existence of preoperative ascites, a rate bilirubin $>1.5 \mathrm{mg} / \mathrm{dL}$ and an albumin level $<30 \mathrm{~g} / \mathrm{l}$. The authors observed, moreover, that the association of a MELD score $>15$ and an albumin level $<25 \mathrm{~g} / \mathrm{l}$ was associated with an increase in the risk of postoperative complications by a coefficient 8 and an increase in the rate of postoperative mortality $(60 \%$ vs. $14 \%, p<0.01)$.

Overall, the existence of cirrhosis classified as Child $\mathrm{C}$ or with a MELD score $>15$ associated with an albumin level $<25 \mathrm{~g} / \mathrm{l}$ is associated with a prohibitive mortality rate and must call into question the surgical indication even if laparoscopic cholecystectomy is technically feasible (rank 4 level of evidence).

\section{Conflict of Interest}

The authors declare no conflicts of interests.

\section{References}

1. Bouchier IA. Postmortem study of the frequency of gallstones in patients with cirrhosis of the liver. Gut. 1969;10(9):705-10. 2. Morino M, Cavuoti G, Miglietta C, Giraudo G, Simone P. Laparoscopic cholecystectomy in cirrhosis: contraindication or privileged indication? Surg Laparosc Endosc Percutan Tech. 2000; 10(6):360-3. 
3. Zhang Y, Liu D, Ma Q, Dang C, Wei W, Chen W. Factors influencing the prevalence of gallstones in liver cirrhosis. J Gastroenterol Hepatol. 2006;21(9):1455-8.

4. Acalovschi M. Gallstones in patients with liver cirrhosis: incidence, etiology, clinical and therapeutical aspects. World J Gastroenterol. 2014;20(23):7277-85

5. Alvaro D, Angelico M, Gandin C, Ginanni Corradini S, Capocaccia L. Physico-chemical factors predisposing to pigment gallstone formation in liver cirrhosis. J Hepatol. 1990;10(2):228-34.

6. Castaing D, Houssin D, Lemoine J, Bismuth H. Surgical management of gallstones in cirrhotic patients. American journal of surgery. 1983;146(3):310-3.

7. Loria P, Lonardo A, Lombardini S, Carulli L, Verrone A, Ganazzi D, et al. Gallstone disease in non-alcoholic fatty liver: prevalence and associated factors. J Gastroenterol Hepatol. 2005;20(8):1176-84.

8. Koller T, Kollerova J, Hlavaty T, Huorka M, Payer J. Cholelithiasis and markers of nonalcoholic fatty liver disease in patients with metabolic risk factors. Scand J Gastroenterol. 2012;47(2):197-203.

9. Poynard T, Lonjon I, Mathurin P, Abella A, Musset D, Bedossa P, et al. Prevalence of cholelithiasis according to alcoholic liver disease: a possible role of apolipoproteins Al and All. Alcohol Clin Exp Res. 1995;19(1):75-80.

10. Barbara L, Sama C, Morselli Labate AM, Taroni F, Rusticali AG, Festi $D$, et al. A population study on the prevalence of gallstone disease: the Sirmione Study. Hepatology. 1987;7(5):913-7.

11. Maggi A, Solenghi D, Panzeri A, Borroni G, Cazzaniga M, Sangiovanni A, et al. Prevalence and incidence of cholelithiasis in patients with liver cirrhosis. Ital J Gastroenterol Hepatol. 1997; 29(4):330-5.

12. Ishizaki $Y$, Bandai $Y$, Shimomura $K$, Shimada $K$, Hashimoto $M$, Sanjyo K, et al. Management of gallstones in cirrhotic patients. Surg Today. 1993;23(1):36-9.

13. Orozco H, Takahashi T, Mercado MA, Prado E, Borunda D. Longterm evolution of asymptomatic cholelithiasis diagnosed during abdominal operations for variceal bleeding in patients with cirrhosis. American journal of surgery. 1994;168(3):232-4

14. Puggioni A, Wong LL. A metaanalysis of laparoscopic cholecystectomy in patients with cirrhosis. J Am Coll Surg. 2003;197(6):921-6.

15. Yokoe M, Takada T, Strasberg SM, Solomkin JS, Mayumi T, Gomi $\mathrm{H}$, et al. TG13 diagnostic criteria and severity grading of acute cholecystitis (with videos). J Hepatobiliary Pancreat Sci. 2013 20(1):35-46.

16. Kiriyama S, Takada T, Strasberg SM, Solomkin JS, Mayumi T, Pitt $\mathrm{HA}$, et al. TG13 guidelines for diagnosis and severity grading of acute cholangitis (with videos). J Hepatobiliary Pancreat Sci. 2013; 20(1):24-34.

17. Laurence JM, Tran PD, Richardson AJ, Pleass HC, Lam VW. Laparoscopic or open cholecystectomy in cirrhosis: a systematic review of outcomes and meta-analysis of randomized trials. HPB (Oxford). 2012;14(3):153-61.

18. de Goede B, Klitsie PJ, Hagen SM, van Kempen BJ, Spronk S, Metselaar HJ, et al. Meta-analysis of laparoscopic versus open cholecystectomy for patients with liver cirrhosis and symptomatic cholecystolithiasis. Br J Surg. 2013;100(2):209-16.

19. Lausten SB, Ibrahim TM, El-Sefi T, Jensen LS, Gesser B, Larsen CG, et al. Systemic and cell-mediated immune response after laparoscopic and open cholecystectomy in patients with chronic liver disease. A randomized, prospective study. Dig Surg. 1999; 16(6):471-7.

20. Ji W, Li LT, Wang ZM, Quan ZF, Chen XR, Li JS. A randomized controlled trial of laparoscopic versus open cholecystectomy in patients with cirrhotic portal hypertension. World J Gastroenterol. 2005;11(16):2513-7.

21. El-Awadi S, El-Nakeeb A, Youssef T, Fikry A, Abd El-Hamed TM, Ghazy $\mathrm{H}$, et al. Laparoscopic versus open cholecystectomy in cirrhotic patients: a prospective randomized study. Int J Surg. 2009; 7(1):66-9.
22. Hamad MA, Thabet M, Badawy A, Mourad F, Abdel-Salam M, Abdel-Rahman Mel T, et al. Laparoscopic versus open cholecystectomy in patients with liver cirrhosis: a prospective, randomized study. J Laparoendosc Adv Surg Tech A. 2010;20(5):405-9.

23. Poggio JL, Rowland CM, Gores GJ, Nagorney DM, Donohue JH. A comparison of laparoscopic and open cholecystectomy in patients with compensated cirrhosis and symptomatic gallstone disease. Surgery. 2000;127(4):405-11.

24. Poniachik J, Castro S, Madrid AM, Quera R, Amat J, Smok G, et al. [Laparoscopic and classic cholecystectomy in patients with liver cirrhosis]. Rev Med Chil. 2002;130(12):1343-8.

25. da Silveira EB. Outcome of cirrhotic patients undergoing cholecystectomy: applying Bayesian analysis in gastroenterology. J Gastroenterol Hepatol. 2006;21(6):958-62.

26. Fernandes NF, Schwesinger WH, Hilsenbeck SG, Gross GW, Bay MK, Sirinek KR, et al. Laparoscopic cholecystectomy and cirrhosis: a case-control study of outcomes. Liver Transpl. 2000;6(3):340-4.

27. Leone N, Garino M, De Paolis P, Pellicano R, Fronda GR, Rizzetto M. Laparoscopic cholecystectomy in cirrhotic patients. Dig Surg. 2001;18(6):449-52.

28. Urban L, Eason GA, ReMine S, Bogard B, Magisano J, Raj P, et al. Laparoscopic cholecystectomy in patients with early cirrhosis. Curr Surg. 2001;58(3):312-5

29. Yeh CN, Chen MF, Jan YY. Laparoscopic cholecystectomy in 226 cirrhotic patients. Experience of a single center in Taiwan. Surgical endoscopy. 2002:16(11):1583-7.

30. Tuech JJ, Pessaux P, Regenet N, Rouge C, Bergamaschi R, Arnaud JP. Laparoscopic cholecystectomy in cirrhotic patients. Surg Laparosc Endosc Percutan Tech. 2002;12(4):227-31.

31. Clark JR, Wills VL, Hunt DR. Cirrhosis and laparoscopic cholecystectomy. Surg Laparosc Endosc Percutan Tech. 2001;11(3):165-9.

32. Cucinotta E, Lazzara S, Melita G. Laparoscopic cholecystectomy in cirrhotic patients. Surgical endoscopy. 2003;17(12):1958-60.

33. Flores Cortes M, Obispo Entrenas A, Docobo Durantez F, Romero Vargas E, Legupin Tubio D, Valera Garcia Z. Laparoscopic treatment of cholelithiasis in cirrhotic patients. Rev Esp Enferm Dig. 2005;97(9):648-53.

34. Curro G, Baccarani U, Adani G, Cucinotta E. Laparoscopic cholecystectomy in patients with mild cirrhosis and symptomatic cholelithiasis. Transplant Proc. 2007;39(5):1471-3.

35. Leandros E, Albanopoulos K, Tsigris C, Archontovasilis F, Panoussopoulos SG, Skalistira M, et al. Laparoscopic cholecystectomy in cirrhotic patients with symptomatic gallstone disease. ANZ J Surg. 2008;78(5):363-5.

36. Mancero JM, D'Albuquerque LA, Gonzalez AM, Larrea FI, de Oliveira e Silva A. Laparoscopic cholecystectomy in cirrhotic patients with symptomatic cholelithiasis: a case-control study. World J Surg. 2008;32(2):267-70.

37. Shaikh AR, Muneer A. Laparoscopic cholecystectomy in cirrhotic patients. JSLS. 2009;13(4):592-6.

38. Pavlidis TE, Symeonidis NG, Psarras K, Skouras C, Kontoulis TM, Ballas $\mathrm{K}$, et al. Laparoscopic cholecystectomy in patients with cirrhosis of the liver and symptomatic cholelithiasis. JSLS. 2009; 13(3):342-5.

39. Delis S, Bakoyiannis A, Madariaga J, Bramis J, Tassopoulos N, Dervenis C. Laparoscopic cholecystectomy in cirrhotic patients: the value of MELD score and Child-Pugh classification in predicting outcome. Surgical endoscopy. 2010;24(2):407-12.

40. Lledo JB, Ibanez JC, Mayor LG, Juan MB. Laparoscopic cholecystectomy and liver cirrhosis. Surg Laparosc Endosc Percutan Tech. 2011;21(6):391-5.

41. Quillin RC, 3rd, Burns JM, Pineda JA, Hanseman D, Rudich SM, Edwards MJ, et al. Laparoscopic cholecystectomy in the cirrhotic patient: predictors of outcome. Surgery. 2013;153(5):634-40.

42. Douard R, Lentschener $C$, Ozier $Y$, Dousset B. Operative risks of digestive surgery in cirrhotic patients. Gastroenterol Clin Biol. 2009;33(6-7):555-64 
43. Bhangui P, Laurent A, Amathieu R, Azoulay D. Assessment of risk for non-hepatic surgery in cirrhotic patients. J Hepatol. 2012; 57(4):874-84

44. de Goede B, Klitsie P, Lange J, Metselaar H, Kazemier G. Morbidity and mortality related to non-hepatic surgery in patients with liver cirrhosis: a systematic review. Best Pract Res Clin Gastroenterol. 2012;26:47-59.

45. Machado NO. Laparoscopic cholecystectomy in cirrhotics. JSLS. 2012;16(3):392-400.

46. Chmielecki DK, Hagopian EJ, Kuo YH, Kuo YL, Davis JM. Laparoscopic cholecystectomy is the preferred approach in cirrhosis: a nationwide, population-based study. HPB (Oxford). 2012; 14(12):848-53

47. Palanivelu C, Rajan PS, Jani K, Shetty AR, Sendhilkumar K, Senthilnathan $\mathrm{P}$, et al. Laparoscopic cholecystectomy in cirrhotic patients: the role of subtotal cholecystectomy and its variants. J Am Coll Surg. 2006;203(2):145-51.

48. Elshaer M, Gravante G, Thomas K, Sorge R, Al-Hamali S, Ebdewi H Subtotal cholecystectomy for "difficult gallbladders": systematic review and meta-analysis. JAMA Surg. 2015;150(2):159-68.

49. Gurusamy KS, Rossi M, Davidson BR. Percutaneous cholecystostomy for high-risk surgical patients with acute calculous cholecystitis. Cochrane Database Syst Rev. 2013;12(8): CD007088.

50. Liu CL, Fan ST, Lo CM, Wong Y, Ng IO, Lam CM, et al. Abdominal drainage after hepatic resection is contraindicated in patients with chronic liver diseases. Ann Surg. 2004;239(2):194-201.

51. Sun HC, Qin LX, Lu L, Wang L, Ye QH, Ren N, et al. Randomized clinical trial of the effects of abdominal drainage after elective hepatectomy using the crushing clamp method. Br J Surg. 2006; 93(4):422-6.

52. Dasari BV, Tan CJ, Gurusamy KS, Martin DJ, Kirk G, McKie L, et al. Surgical versus endoscopic treatment of bile duct stones. Cochrane Database Syst Rev. 2013;12(12):CD003327.

53. Adler DG, Haseeb A, Francis G, Kistler CA, Kaplan J, Ghumman SS, et al. Efficacy and safety of therapeutic ERCP in patients with cirrhosis: a large multicenter study. Gastrointest Endosc. 2016;83(2):353-9.

54. Qiu J, Yuan H, Chen S, Wu H. Laparoscopic common bile duct exploration in cirrhotic patients with choledocholithiasis. Surg Laparosc Endosc Percutan Tech. 2015;25(1):64-8.

55. Wu H, Giu JG, Yuan HC, Chen ST. Laparoscopic common bile duct exploration in cirrhotic patients with choledocholithiasis: Retraction. Surg Laparosc Endosc Percutan Tech. 2015;25(2):184. 56. Pawlik TM, Olbrecht VA, Pitt HA, Gleisner AL, Choti MA, Schulick $\mathrm{RD}$, et al. Primary sclerosing cholangitis: role of extrahepatic biliary resection. J Am Coll Surg. 2008;206(5):822-30; discussion 30-2.

57. Telem DA, Schiano T, Goldstone R, Han DK, Buch KE, Chin EH, et al. Factors that predict outcome of abdominal operations in patients with advanced cirrhosis. Clin Gastroenterol Hepatol. 2010; 8(5):451-7, quiz e58. 